\title{
CONFERENCIA MAGISTRAL
}

\section{COMISIÓN NACIONAL DE BIOÉTICA. ASIGNATURA PENDIENTE EN URUGUAY ${ }^{1}$}

Mariana Blengio Valdés*

En Uruguay la creación de una Comisión Nacional de Bioética constituye una asignatura pendiente en la agenda de la bioética y los derechos humanos. Si bien no existe un modelo único ni ideal de Comisión Nacional, la experiencia de las Comisiones de esta naturaleza creadas en Europa y América Latina ilustran su relevancia y proyectan sus funciones. El compromiso de los Estados de crearlas y fortalecerlas emana de documentos claves como la trilogía de declaraciones adoptadas por la Conferencia General de la UNESCO en 1997, 2003 y 2005. En tal sentido la última de las tres, concretamente la Declaración de Bioética y Derechos Humanos exhorta a los Estados Partes a su creación como herramientas de fortalecimiento de la democracia en el marco de la importancia de la discusión y reflexión sobre grandes problemas que vinculan a la vida y salud humanas en su conexión con la naturaleza, a la luz de impacto de los avances biotecnológicos y biomédicos. A lo cual se suman en el inicio de una nueva década, las circunstancias que en este siglo XXI parecen interpelar las relaciones humanas a partir del impacto de una pandemia con consecuencias devastadoras en lo sanitario, social y económico.

\section{Punto de partida. La Bioética como disciplina.}

El estudio sistemático de la conducta humana en el campo de las ciencias biológicas y la atención de la salud constituye el campo de estudio de la Bioética, concebida ésta como una disciplina que examina dichos aspectos a la luz de los valores y principios morales.(REICH, W.T, 1995. Enciclopedia de Bioética).

El desarrollo de la Bioética se nutre de un proceso progresivo de incorporación de corrientes de pensamiento. Todas éstas interactúan en la reflexión abierta, pluridisciplinaria y plural. Este proceso se impulsará en las últimas décadas del siglo XX y especialmente el siglo XXI a partir de la puesta en común de fuertes dilemas que se hacen eminentemente

\footnotetext{
*Doctora en Derecho y Ciencias Sociales. Profesora (agr) en Derechos Humanos y Bioética UDELAR, CLAEH y CEJU. Directora INDDHH y Defensoría del Pueblo. Coordinadora de la Cátedra UNESCO de DDHH de la UDELAR (2002 - 2016). Integrante de las Comisiones de Bioética y Salud Asistencia del MSP (2011); Comisión de Reproducción Humana Asistida ley 19167 (2016 - 2017 ), Comité de Investigación en seres humanos Facultad de Medicina UDELAR (2016 Y 2017), Comisión de Bioética del CMU (2020/21). Fundadora del Grupo de Derecho y Bioética de la Facultad de Derecho UDELAR. Miembro del Grupo de Mujeres Constitucionalistas Facultad de Derecho UDELAR. Autora de diversas obras en su especialización. Correo electrónico: ius@netgate.com.uy

${ }^{1}$ Texto revisado por la autora de la conferencia dictada en el ciclo de talleres y conferencias de educación continua organizados por el Colegio Médico del Uruguay el 28 de abril 2021.
} 
Ya adentrado el siglo XXI, el campo de estudio de la Bioética se amplía sustancialmente. A los abordajes relativos a la atención de la salud y las ciencias médicas se une la necesidad de reflexionar, analizar y cuidar la supervivencia del ser humano, los demás seres vivos y el entorno vital. Se incluyen además de la ética clínica y la investigación científica con seres humanos; aspectos vinculados a la administración de recursos ambientales; derecho de los seres vivos no humanos; ecologismo; ciencias cognitivas; genética; inteligencia artificial; big data; derechos de las generaciones futuras; ciencia y sociedad; entre otros puntos.

En el estudio de la Bioética se pone en relación el conocimiento del mundo biológico con la formación de actitudes y políticas públicas encaminadas a conseguir el bien social a la luz del ininterrumpido y constante progreso de la biomedicina y la biotecnología. Los avances científicos a la vez que constituyen esperanzas para múltiples grupos humanos generan profundos dilemas individuales y colectivos.

\section{Reflexión como esencia de la Bioética.}

El objetivo de la Bioética es reflexionar en torno al fenómeno de la vida en general y la salud en particular y los problemas que se suscitan desde una visión amplia e integral. Esta reflexión promueve la búsqueda y logro de compromisos mínimos en la elaboración de reglas de juego que puedan ser aceptadas por los individuos que conviven en las diferentes comunidades. Promoviéndose su participación como proceso imprescindible para la adopción normas jurídicas que contengan un mínimo consenso sobre valores individuales y colectivos.

La reflexión parte de considerar que "la Bioética está precisamente en el reconocimiento de la pluralidad de opciones morales y en propugnar la necesidad de establecer un mínimo marco de acuerdo por medio del cual los individuos pertenecientes a comunidades morales diversas puedan considerarse ligados por una estructura común que permita la resolución de los conflictos con el suficiente grado de acuerdo". Por último, "será el Derecho el que deberá establecer los límites de lo permitido, de ahí que deriva la estrecha relación entre Derecho y Bioética”. (CASADO,M.2016).

La Bioética no impone, porque sus propuestas de tratamiento de los conflictos solo pueden ser exigibles en caso de pasar a ser reglas jurídicas. Es una disciplina que en tanto rama de la ética y ésta de la filosofía, tiene como función ofrecer criterios o pautas generales para orientar la acción humana. Esto implica la necesidad de elaborar sólidos argumentos que se conviertan en razones para la acción propia o ajena, de cara a la toma 
de decisiones ya sean particulares o generales como es el caso de la elaboración de las normas jurídicas.

\section{Consenso internacional en la proyección del Derecho Internacional de la Bioética y sus principios.}

El desarrollo de la Bioética como disciplina y la consolidación de los principios bioéticos como disposiciones abiertas que permiten argumentar y dirimir los grandes dilemas existenciales se potencia a finales del siglo XX y comienzos del siglo XXI. Esto se produce al llegar a consensos mínimos que en esencia argumentan que: el respeto al valor de la dignidad humana es condición de la existencia misma de la vida; que ciertos límites no pueden ser trasgredidos; que los criterios de pura eficacia técnica deben rechazarse en la medida que no contemplen los aspectos éticos.

La comunidad internacional ha sido partícipe de este proceso especialmente a partir del fin de la segunda guerra mundial y a la luz de la violación flagrante de los derechos humanos verificada en este período incluyendo la experimentación con seres humanos. Lo que no obsta a la constatación de otros múltiples abusos a partir de investigaciones con seres humanos en el seno de diferentes Estados, antes, durante y después de la II Gran Guerra, que tomarán estado público ya adentrado el siglo XX.

En el período indicado documentos claves son aprobados por la comunidad internacional, incluyendo disposiciones que fundadas en el respeto de la dignidad de los seres humanos apuestan a erradicar la vulneración de los derechos y el reconocimiento de la autonomía de la persona en relación a su vida, su salud, y su entorno. La Declaración Universal de los Derechos Humanos adoptada en 1948², el Pacto de Derechos Civiles y Políticos de 1966 específicamente su artículo $7^{3}$ y el Pacto de Derechos Económicos Sociales y Culturales en el artículo $15.3^{4}$, son ejemplos de ello y abren las puertas a un desarrollo normativo de acuerdos internacionales que constituyen el núcleo duro de la protección universal y su debido equilibro en materia de derechos y libertades. Estos documentos generan el entronque entre el Derecho Internacional de los Derechos Humanos y el de la Bioética.

\footnotetext{
${ }^{2}$ Adoptada en 1948 incorpora en su preámbulo el reconocimiento a la dignidad de la persona destacando que "el desconocimiento y menosprecio de los derechos hu7anos han originado actos de barbarie ultrajantes par la conciencia de la humanidad".

${ }^{3}$ PDCP adoptado por ONU en 1966. Artículo 7. Nadie será sometido sin su libre consentimiento a experimentos médicos o científicos. Ratificado por ley 13751 de 11 julio 1969.

${ }^{4}$ PDESC adoptado por ONU en 1966. Artículo 15.3. Los Estado Partes reconocen los beneficios que derivan del fomento y desarrollo de la investigación científica y para la sociedad creadora. Ratificado por ley 13751 de 11 julio 1969.
} 
En ese marco surgen desde ese período y en forma progresiva normas que incorporarán paulatinamente los que hoy se consolidan como principios bioéticos.

El Código de Nuremberg en 19475; la Declaración de Helsinski de 1964 y sus sucesivas modificaciones ${ }^{6}$; las Pautas Eticas Internacionales para la Investigación relacionada con la Salud con Seres Humanos elaboradas por el Consejo de Organizaciones Internacionales de las Ciencias Médicas (CIOMS) con la colaboración de la OMS sucesivamente revisadas ${ }^{7}$, son también ilustrativas. Lo que arroja un denominador común de disposiciones abocadas a proteger y respetar el valor de la dignidad humana más allá de la naturaleza jurídica del documento y la entidad que lo emitió.

El proceso se cierra en esta etapa de consolidación de la Bioética con la trilogía de declaraciones adoptadas por la Conferencia General de la Unesco en 1997, 2003 y 2005. Estas son la Declaración Universal sobre Genoma Humano y Derechos Humanos ${ }^{8}$; la Declaración Internacional sobre Datos Genéticos y la Declaración Universal sobre Bioética y Derechos Humanos (en adelante DUBDDHH). Documentos que abordan la esencia de múltiples aspectos sustanciales enumerando principios bioéticos que emergen del respeto a la dignidad humana, e incluyen la creación y proyección de ámbitos de reflexión en el ámbito de las Naciones Unidas como lo constituyen el Comité Internacional de Bioética (CIB) y el Comité Intergubernamental Bioética.

La Declaración Universal de Bioética y Derechos Humanos ${ }^{9}$ (en adelante DUBDDHH) reúne a partir del principio del respeto a la dignidad humana el conjunto de principios bioéticos que proyectarán la reflexión y la búsqueda de acuerdos y consensos mínimos en el abordaje de temas sustanciales para la especie humana. Su adopción es considerada sustancial en la historia de la protección universal de los derechos humanos en un entronque con el derecho internacional de la Bioética que permitirá proyectar los principios bioéticos en ella enumerados.

\footnotetext{
${ }^{5}$ Publicado en 1947 surge a partir del juicio llevado adelante para juzgar los experimentos con seres humanos llevados a cabo por médicos ("Doctores") durante la segunda guerra mundial en campos de concentración nazis. Es una primera forma jurídica de establecer pautas que protejan a los sujetos de investigación. Centra sus disposiciones en el consentimiento informado.

${ }^{6}$ Adoptada por la Asamblea de la Asociación Médica Mundial en Finlandia. Comprende principios éticos para las investigaciones con seres humanos. Ha sido objeto de sucesivas modificaciones.

${ }^{7}$ Disponibles en: https://cioms.ch/wp-content/uploads/2017/12/CIOMS-EthicalGuideline_SP_INTERIOR-FINAL.pdf Fecha de consulta: 4 febrero 2021.

${ }^{8}$ Esta Declaración adoptada en 1997 por la Conferencia General de UNESCO fue hecha suya en 1998 por la Asamblea General de la ONU adoptándose por unanimidad en ambas instancias.

9 Puede ampliarse en: BLENGIO VALDÉS, Mariana. La dignidad humana como parámetro de interpretación en fuentes de Derecho Internacional de los DDHH y la Bioética. Revista de Derecho Público, 2016. Disponible en: http://www.revistaderechopublico.com. uy/revistas/49/archivos/Blengio49.pdf Fecha de consulta: 7 febrero 2021.
} 
A este desarrollo se unen múltiples normativas incorporadas a nivel nacional que abordan temas objeto de la bioética en una gran cantidad de Estados así como también la creación de ámbitos de reflexión en comités o comisiones de carácter temporario o permanente que pretenden dar un marco teórico al impacto que la biotecnología y la biomedicina produce en la vida humana. ${ }^{10}$

\section{La DUBDDHH y las Comisiones Nacionales de Bioética}

Adoptada en 2005 la Declaración Universal de Bioética y Derechos Humanos fue adoptada por unanimidad (téngase presente que en 1948 la Declaración Universal de Derechos Humanos observó abstenciones) lo que resulta claramente significativo por la relevancia de los principios que incorpora. Con especial referencia a la temática abordada en este aporte, la DUBDDHH exhorta a los Estados Partes a crear comisiones nacionales de Bioética como herramientas de fortalecimiento de la democracia en el marco de la relevancia de la discusión de los grandes problemas existenciales. Su artículo 19 dispone:

Su artículo 19 insta a crear, promover y apoyar la creación y labor de comités de ética independientes y pluridisciplinarios. Enumera una tipología de comités de ética necesaria para la aplicación de los principios contenidos en la DUBDDHH. Entre ellos los comités de ética de investigación clínica, los comités de ética asistencial y las comisiones nacionales de bioética. Las tres categorías comprenden perspectivas y cometidos diferentes en su especialidad y ámbito de acción. En forma adicional el artículo 22.2 dispone que los: "Estados deben fomentar la creación de comités de ética independientes, pluridisciplinares y pluralistas". Se insta de esa forma a crear instancias de reflexión ética y a que los estados lo potencien. ${ }^{11}$

Las comisiones nacionales de bioética tienen como esencia la aplicación de los principios de la DUBDDHH cuyo objetivo es tratar las cuestiones éticas relacionadas con la medicina, las ciencias de la vida y las tecnologías conexas aplicadas a los serse humanos, teniendo en cuenta las dimensiones sociales, jurídicas y ambientales (artículo $1)$.

\footnotetext{
${ }^{10}$ La creación en Estados Unidos de la Comisión Nacional para la Protección de Seres Humanos en 1974 fue un intento del gobierno de dicho país por proyectar un abordaje que permitiera instaurar determinadas pautas básicas en el ámbito de la investigación en seres humanos ante el escándalo público generado por los estudios experimentales con humanos en Willowbrock y Tuskiggee realizados con grupos especialmente vulnerables, sin ningún tipo de contemplación de sus derechos y con la utilización de fondos públicos. La Comisión que actuara durante 4 años (1974 - 1978) emitió su Informe sobre Principios Eticos y Pautas de Protección de los Seres Humanos de Investigación - Infome Belmont basado en cuatro principios bioéticos: autonomía, beneficencia, no maleficencia y justicia. Este documento tendrá una amplia repercusión y su proyección universal para el estudio y aplicación práctica de la Bioética. ${ }^{11} \mathrm{Si}$ bien la creación de estos ámbitos ya había sido recomendada en las anteriores declaraciones de la UNESCO, concretamente en el artículo 16 de la Declaración sobre Genoma Humano y DDHH de 1997 y el artículo 6 b de la Declaración Internacional de Datos Genéticos de 2003, por primera vez se definen las funciones que cada uno debe desempeñar en diferentes contextos.
} 


\section{Fundamentos e importancia de las Comisiones Nacionales de B ioética.}

En la medida que los avances científicos y sus aplicaciones pueden incidir en el efectivo goce de los derechos humanos de todas las personas y muy especialmente en el ámbito clínico y de investigación, es que se considera que las comisiones nacionales de bioética en carácter de foro de reflexión ética contribuyen a garantizar y proteger los mismos. Aunque sus informes y actuaciones no se reduzcan a temas de ética clínica o investigación. A juicio de algunos autores estos foros se visualizan como verdaderos “trampolines para la reflexión ética en un Estado" (DE LECUONA, I., 2015).

Su creación, la cual se plasma en el ordenamiento jurídico a través de disposiciones de diferente jerarquía, ilustra claramente el vínculo entre ética y derecho. Están abocadas a contribuir al debate social y a la cohesión de la sociedad civil en sus distintas esferas. "Su regulación nos ayuda a disponer de un marco normativo adecuado que pueda dar respuesta a los nuevos retos científicos al mismo tiempo que garantice la protección de los derecho s delas personas que pudiesen resultar afectados por la acción investigadora”. En muchos casos apuestan a "generar confianza entre la sociedad y los investigadores, para encontrar el equilibrio entre el respeto por los derechos humanos como puede ser la intimidad el libre desarrollo de la personalidad y la libertad de investigación. (DE LECUONA, I., 2015)

Las Comisiones Nacionales de Bioética abordan temas muy complejos y en muchos casos novedosos pero ligados a lo cotidiano y lo ordinario que deriva en esencia de la investigación biomédica y sus aplicaciones. Así también de cuestiones éticamente arduas que promueven una discusión social de especial interés. Por eso es que se justifica y valora con creces, la creación de estos ámbitos de reflexión. El vertiginoso avance científico interpela al ser humano con preguntas cada vez mas fundamentales sobre la esencia misma de la vida.

La creación de las Comisiones Nacionales de Bioética como ámbitos de reflexión y con características propias que las identifican, cumplen una función especializada y complementaria del debate parlamentario pero no son comisiones parlamentarias ni tienen como cometido representar a los parlamentarios.

Por su parte no emiten normas vinculantes. Deben ser sus dictámenes insumos para la discusión, "brújulas" que pueden orientar al momento de tomar decisiones tanto en el ámbito judicial como a la hora de legislar. Por eso su composición debe incluir la mayor cantidad de enfoques y perspectivas que aseguren un discurso bioético público buscando el debido y variado perfil de sus integrantes. (HERRANZ, G, 2005). 
El "rigor lógico, el conocimiento profundo y el juicio sólido" son tres de las cualidades básicas que deben tener los integrantes de este tipo de Comisiones Nacionales lo que se habrá de reflejar en los informes a emitir. Las conclusiones a las cuales se arriban no pueden dejar vacíos lógicos excluyendo cualquier tipo de argumento basado en la autoridad o el dogma. La mayoría de la doctrina es unánime en afirmar que el partir de la reflexión como eje de la actuación; la independencia de criterio; y las razones fundadas; constituyen los componentes más poderosos de este tipo de órganos y de los documentos que emiten. A los cuales solo se arribará en la medida que los integrantes de las comisiones nacionales de bioética observen estos elementos y se incorporen desde la pluridisciplinariedad y la pluralidad. La ética se aconseja no se impone como obligación. No debe ser un poder fáctico sino desde el argumento lógico, extraer la fuerza moral. "Su ascendiente está en la objetividad ponderada con que recopila los datos. La ecuanimidad con que orden a los argumentos, la consistencia con la cual ordena sus conclusiones y las envía a la sociedad. No son un legislativo paralelo" (HERRANZ, G, 2005).

\section{Antecedentes europeos y americanos.}

Es en Europa donde se crean las primeras comisiones nacionales de bioética ya desde la década del 80. Esto arroja más de 40 años de experiencia. Así puede verse el caso de Inglaterra (1981 y 2000); Dinamarca en 1982, Francia en 1983. El Comité National Consultatif d'Ethique pour les Sciences de la Vie et de la Santé creado en Francia reúne 36 miembros siendo un ejemplo de los más numerosos. Es en la década del 90 donde se crearán otras comisiones nacionales: Italia, Portugal, Holanda, Bélgica y Suiza. Su regulación y forma de actuación así como composición es diversa. Sin perjuicio de ello todas se basan en ese denominador común antes referenciado de reflexión independiente, plural y pluralista de la realidad humana y sus dilemas éticos.

Aunque posterior el proceso en América Latina es también constante. La primera fue Cuba en 1997, verificándose su creación en Haití en 1999; en Bolivia 2013; México 2005, Ecuador 2012, El Salvador 2009, Jamaica 2009, República Dominicana 2009, Paraguay 2017, estando en proceso Colombia y Brasil. De los 12 países que las han creado 2 han sido dispuestas por normas legislativas y el resto por resoluciones ministeriales.

\section{Uruguay.}

En Uruguay la creación de una Comisión Nacional de Bioética con las características que acabamos de delinear constituye como se expresó, una asignatura pendiente en la agenda de la bioética y los derechos humanos.

Discusiones sobre su pertinencia y rol se vienen verificando desde fines de la década del 90. A la fecha ninguna comisión ha sido creada en tal carácter y con las 
particularidades reseñadas y tampoco ha asumido el rol que se describe en este aporte. El tema forma parte de los desafíos que posiciona la academia como elemento relevante para la proyección de la bioética, beneficiando a la sociedad en su conjunto y la democracia.

En lo que refiere a la temática de la Bioética en general, desde el Poder Ejecutivo en las décadas del 90 y comienzos del siglo XX se constata un destacado rol que en el ámbito internacional observó Uruguay como Estado Parte de la Conferencia General de la UNESCO en la discusión y posterior adopción de la trilogía de las declaraciones antes referenciadas. Así también en la participación de Uruguay a través de especialistas y representantes gubernamentales en el seno de los Comités Internacional e Intergubernamental de Bioética.

Por su parte en el ámbito interno, la creación y puesta en marcha de comités de bioética y otros con cometidos específicos referidos a la ética en investigación con seres humanos en diversos centros asistenciales, avalan no solo la relevancia que la academia da a estas instancias de reflexión sino también ilustran el interés que profesionales, especialistas y asociaciones científicas de diversas disciplinas han marcado en este terreno. A modo de ejemplo a partir de la década del 90 se crean las Comisiones de Bioética en el Sindicato Médico del Uruguay (SMU), Hospital Pereira Rossell, Hospital de Clínicas, CASMU, Hospital Policial, Hospital Maciel, Círculo Católico. Téngase presente que a partir del año 2008 con la aprobación de la ley 18335 la creación de comisiones de bioética en el ámbito asistencial son obligatorias para todo el sistema nacional integrado de salud incluyendo la obligatoriedad en las emergencias móviles incorporadas luego por decreto 274/2010. Por Resolución 610/2005 el MSP creó la Comisión de Bioética y Calidad Integral en la Atención de la Salud en el ámbito de dicho Ministerio.

En lo que refiere a la ética de investigación con seres humanos no se registra a la fecha una ley específica que regule la temática y todas sus aristas, en forma integrada lo que reiteradamente hemos señalado y considerado un tema pendiente. Co existen en materia legislativa diferentes disposiciones que refieren a la temática en leyes dispersas como las previstas en las leyes de reproducción humana asistida, donación y trasplantes, entre otras. ${ }^{12}$ Con aspectos no menores que vinculan a la investigación con embriones, células madres y demás. La regulación legal en forma integrada, constituye un vacío que deberá oportunamente abordar el legislador para otorgar al Uruguay una normativa de rango legal que apueste a la regulación de la temática cuya actualidad resulta sustancial máxime a la luz del impacto de la pandemia en 2019 - 2020.

\footnotetext{
${ }^{12}$ Entre las diferentes leyes que refieren a aspectos puntuales de la investigación con seres humanos encontramos: Ley 18968 Donación y Trasplantes de Organos células y tejidos; ley 19167 de Reproducción Humana Asistida; Ley 19268 Código de Etica Médica; Ley 18611 sobre utilización de animales en investigaciones científicas. Sobre el punto nos remitimos al texto de nuestra autoría titulado "Sobre la regulación jurídica bioética de la investigación en seres humanos" disponible en: http://www.revistaderechopublico. com.uy/revistas/50/blengio.php 4 febrero 2021.
} 
Sin perjuicio de esto téngase presente que el decreto 158/2019 del Poder Ejecutivo de 3 de junio 2019 al cual nos remitimos, modificativo del decreto 379/2008 que originalmente reguló la temática de investigación en seres humanos incorpora una amplia regulación del tema. Este decreto además de ampliar definiciones y aspectos instrumentales, actualizó la actuación de una Comisión Nacional de Etica en Investigación en Seres Humanos la cual se encuentra vinculada a la Dirección de Salud del MSP donde funciona. Dicha Comisión de carácter pluridisciplinario, "debe velar por el buen funcionamiento de los comité de ética de investigación institucionales". Estos comités de ética en investigación institucionales cumplen un rol específico. Deben revisar y aprobar los protocolos de investigación que se presenten en cada institución e involucren seres humanos. Les corresponde a estos comités institucionales, ponderar los aspectos metodológicos, éticos y legales del protocolo así como balance de riesgos y beneficios emitiendo su aprobación, observación o no aprobación la que será preceptiva y vinculante (artículo 38). En el caso de los estudios multi céntricos que requieran dictamen único; poblaciones vulnerables; aspectos de bioseguridad y aquellos ensayos clínicos de nuevos medicamentos, test, vacunas, equipamientos, dispositivos y procedimientos invasivos" será la Comisión Nacional de Etica en Investigación que funciona en el ámbito del MSP la que deba "dictaminar del punto de vista ético sobre los mismos (artículo 43). Resulta importante no confundir y especificar claramente las funciones y sus consecuencias en relación al carácter preceptivo y vinculante o no de los dictámenes de los comités de ética en investigación con seres humanos institucionales incluyendo a la comisión nacional que funciona en el ámbito del MSP, lo que no obsta a su importancia aspecto que destacamos, y en forma coincidente entendemos que el legislador deberá abordar en el ámbito legislativo, para fortalecer la protección de los seres humanos en su carácter de "sujetos" y no "objetos" de investigación.

\section{Uruguay y la discusión en el ámbito legislativo.}

El Poder Legislativo no ha sido ajeno a la posibilidad de crear una Comisión Nacional de Bioética. Según consta en los antecedentes parlamentarios en 1995 un Grupo Interdisciplinario de representantes nacionales participó en la 93 . Conferencia de la Unión Interparlamentaria realizada en España donde se abordó el tema de las Comisiones Nacionales de Bioética y en el cual se exhortó a establecer este tipo de comisiones en sus respectivos países.. ${ }^{13}$

\footnotetext{
${ }^{13}$ En esa fecha el entonces diputado Jaime Trobo en informe brindado en la Cámara de Representantes hizo referencia a dicha participación destacando que fueron discutidos temas relativos a las implicaciones de la Bioética a nivel mundial para la protección de los derechos humanos. A partir de esta intervención y antecedente se resolvió crear una Comisión Especial sobre Bioética integrada por 10 miembros para el estudio del proyecto que disponga la creación de una Comisión Nacional de BioéticaDisponible en: https:// parlamento.gub.uy/documentosyleyes/ficha-asunto/1521 Fecha de consulta: 6 de febrero 2021.
} 
En el año 2000 legisladores del Partido Nacional presentaron un proyecto conjunto de creación de una Comisión Nacional de Bioética ${ }^{14}$.

En 2003 basado en estos proyectos la Cámara de Representantes elaboró un proyecto de ley por el cual se crea la Comisión Nacional de Bioética ${ }^{15}$. El mismo fue suscrito por los representantes Raúl Argenzio, Gustavo Vachetti, Daniel Bianchi, José Gallo, Orlando Gil, Ramón Legnani y Wilmer Trivel. El mismo se aprueba en 2003 en el plenario de la Cámara y debido a los tiempos legislativos no fue tratado por el Senado y se archiva.

En 2006 el proyecto se desarchiva por la Comisión de Salud Pública para la nueva Legislatura (2005 - 2010) ${ }^{16}$ y se vuelve a presentar suscrito por los representantes José Gallo, Miguel Asqueta, José Quintín Olano y Alvaro Vega. Se aprueba por la Cámara de Representantes el 12 de julio de 2006 y pasa al Senado. El 28 de julio entra en Senado y el 31 de julio pasa a la Comisión de Salud Pública de dicha Cámara. El 1 de agosto se hace distribuido en Comisión de Salud Pública del Senado dándose cuenta en el seno de la Comisión sin verificarse más movimientos. En el año 2010, el 14 de febrero se solicita el archivo. ${ }^{17}$

Desde 2006 a la fecha no ha habido más proyectos de ley sobre la creación de una Comisión Nacional de Bioética lo que no obsta a que el tema se encontrara en la agenda de los desafíos bioéticos tal cual emerge de múltiples actividades realizadas en el ámbito académico y profesional e incluso en muchas instancias con participación parlamentaria. A modo de ejemplo en el marco del apoyo brindado a Uruguay por el Programa ABC de la UNESCO abocado a promover la creación de comisiones nacionales e implementado desde la Oficina Regional con sede en nuestro país, en 2017 se llevó a cabo el Tercer Seminario Regional de Bioética y Etica de la Ciencia para América Latina y el Caribe y el Tercer Seminario Regional de Comités Nacionales de Bioética. En la instancia participaron representantes de 16 países y autoridades nacionales de Uruguay, entre ellos los entonces Ministros de Salud Pública y Vivienda Medio Ambiente y Ordenamiento Territorial, Jorge Basso y Eneida de León. Entre los objetivos de dicho encuentro se

\footnotetext{
${ }^{14} \mathrm{El}$ mismo fue suscrito por los representantes nacionales Beatríz Argimón, Roberto Arrarte, Ricardo Berois, Gustavo Borsari, Julio Cardozo, Jorge Chapper, Francisco Gallinal, Carlos González, Arturo Heber, Lusi Alberto Lacalle, Luis Leglise, Julio Lara, Francisco Ortiz, Gustavo Penades, Alberto Perdomo, María Rivero, Ambrosio Rodríguez, Julio Silveira, Jaime Trobo y Carmelo Viladín. En la exposición de motivos se reconoce la labor en la materia llevada adelante por el especialista Héctor Gros Espiell, quien en tal carácter y por designación directa del Secretario General de la UNESCO en dos períodos consecutivos integró el Comité Internacional de Bioética, donde se gestarían las declaraciones de la Unesco ya referenciadas. También en ese año se presentó un proyecto presentado por Alberto Scavarelli del Partido Colorado.

Puede verse en: Comisión de Salud Pública y Asistencia Social. Carpeta No. 50 de 2000. Repartido No. 21. Marzo 2000

${ }^{15}$ Comisión de Salud Pública y Asistencia Social. Carpeta No. 50 marzo 2000. Anexo I al Repartido 21 de octubre 2003.

${ }^{16}$ Comisión de Salud Pública y Asistencia Social. Carpeta No. 50 de 2000. Anexo 1 al repartido No. 209 junio de 2006.

${ }^{17}$ Disponible en: https://parlamento.gub.uy/documentosyleyes/ficha-asunto/13452/ficha_completa Fecha de consulta: 6 febrero 2021.
} 
encontraba el fortalecimiento de las Comisiones Nacionales de Bioética ya existentes, y en el caso de Uruguay y otros países que aún no la tuvieran, su creación, intentándose identificar retos y desafíos para su concreción. ${ }^{18}$

En la agenda parlamentaria si bien no se retoma la creación de una Comisión Nacional de Bioética, si aparecen disposiciones legislativas con aspectos vinculados a la bioética y la ética de la investigación en seres humanos. Desde la ley 18211 de creación del Sistema Nacional Integrado de Salud que remite a la observancia de los principios bioéticos; a normas que consagran los derechos y obligaciones de pacientes y usuarios en el año 2008 (ley 18335) hasta otras de carácter específico como ser: derechos sexuales y reproductivos 2008 (ley 18426); voluntades anticipadas 2009 (ley 18473); Trasplantes y Donación de Organos 2012 (ley 18968); interrupción del embarazo 2012 (ley 18987), Reproducción Humana Asistida 2013 (Ley 19167); Código de Etica Médica 2014 (Ley 19286); Salud Mental 2017 (19529) entre las más sustanciales.

\section{Aspectos básicos a incluir en su regulación legal.}

Lo importante de su creación radica en la comprensión de que su carácter nacional e independiente la ha de erigir y la debería jerarquizar como un ámbito de reflexión cuyo objetivo radica en consolidarse como puente de abordaje de los grandes problemas de la bioética. No para dar soluciones únicas en tanto su gran desafío es consensuar las diferencias y su prestigio público se consolida con la sabiduría, oportunidad y solidez de sus informes, sino para ilustrar con toda su actuación, las distintas perspectivas humanas que confluyen en todos los desafíos bioéticos.

Sus principios rectores en armonía con la DUBDDHH, han de ser el pluralismo, la participación, la independencia y la trasparencia.

La independencia se erige como garantía indispensable para que puedan desplegarse opiniones libres de cualquier amenaza de coacción o influencia indebida. Tanto externas, por lo cual en ningún caso puede tener dependencia funcional o interna, eliminando jerarquías y dependencia laboral entre sus miembros. Así como también conflictos de interés y destacando su carácter honorario lo que no obsta a fortalecer las funciones ejecutivas de algún consejero y la debida logística y administración con una sede y presupuesto que le permita actuar con dicha independencia. ${ }^{19}$

\footnotetext{
${ }^{18}$ Los Comités y las Comisiones Nacionales de Bioética en América Latina y El Caribe. (Maglio, Ignacio Coordinador). Unesco. Universidad del Bosque. 2018.

${ }^{19}$ Vé nota 6.
} 
El pluralismo supone que deba observarse una representación de todos los sectores tanto del ámbito gubernamental como de la sociedad civil que contenga la pluralidad de creencias sentimientos y convicciones y asegure una acción pluralista.

"Las CNB funcionan como órganos democráticos en los que se promueven de manera deliberativa posiciones morales distintas diferentes intereses pensamientos no unívocos, diversidad de costumbres, y subjetividades que reivindican los derechos de los individuos ante el malestar que les suscita no acceder de igual forma a los medios disponibles. Exige comprender la sociedad de nuestro tiempo y como se expresa, sus contradicciones, la recreación de las urbes en si mismo". (OVALLE GOMEZ, C. 2018).

En cuanto a la pluridisciplinariedad se hace hincapié en la articulación de los saberes, prácticas y disciplinas vinculadas a la ética. Con abordaje integral de nuevos fenómenos de estudio: medio ambiente, biodiversidad, erradicación de la pobreza multiculturalismo entre otros. "Evitar la supremacía de un solo saber o disciplina en particular medicina o biomedicina resabio de modelo hegemónico que subalterniza otras prácticas y saberes”. (HERRANZ, G, 2005).

Sobre su integración, no existe una fórmula o modelo ideal. Si puede destacarse uno de sus elementos: la heterogeneidad de la composición social y la disparidad de intereses.

Los principios enumerados sientan las bases del debate democrático, plural e interdisciplinario sobre los problemas éticos que emergen de la vida humana y no humana en sus propios contextos, en concordancia con sus dimensiones histórico - sociales y medioambientales. Siendo a la vez sustancial que la Comision adopte el enfoque de los derechos humanos de la DUBDDHH para el abordaje de los problemas bioéticos. Entendiendo que dicho marco otorga la referencia fundamental para dar cabida a una visión sobre la complejidad de los temas éticos relacionados con la vida en general y la salud en particular.

La Comisión Nacional de Bioética de un país debe asentarse como una herramienta potente del Estado para contribuir en calidad de insumo teórico práctico, aportando posibles elementos para el futuro diseño de políticas públicas y prácticas gubernamentales que trasciendan las sucesivas administraciones. Su regulación legal proyecta y asienta su creación en el tiempo y la fortalece en su carácter nacional e independiente, más allá de las administraciones. 


\section{Aspectos relevantes a incluir en un proyecto de ley de creación de una Comisión Nacional de Bioética.}

A continuación se especificarán los aspectos básicos que se entiende pertinentes y necesarios incluir en una futura legislación sobre la temática abordada.

Sobre su creación por vía legal. La creación legal aporta permanencia y fortaleza. $\mathrm{Su}$ carácter independiente, pluridisciplinario y plural proyectan su creación en forma acorde con la Constitución y el Derecho Internacional de los Derechos Humanos y la Bioética. Insistimos con su carácter independiente, pluridisciplinario y plural en el marco de lo establecido en la Declaración Universal de Bioética y Derechos Humanos de 2005 y en observancia de la Constitución y los tratados internacionales de derechos humanos suscritos por la República.

Sobre sus competencias: Basándonos en la premisa del asesoramiento la propuesta refiere a los grandes campos en los cuales debe atribuirse su competencia. Se consideran sustanciales los aspectos que se detallan a continuación. Este punteo se realiza tomando como referencia ejemplos de otras regulaciones de América Latina y El Caribe que aportan un contexto amplio y a la vez detallado de las cuestiones a abordar y su forma.

a. Elaborar estudios y dictámenes acerca de conflictos éticos existentes en el campo de la salud o que tengan importancia para la preservación de la vida humana, para la relación del ser humano con el medio ambiente y el acceso a los progresos y avances del conocimiento en las áreas de la salud, la biología y la medicina.

b. Emitir recomendaciones sobre las implicancias morales y éticas de cuestiones emergentes o persistentes que tenga o puedan tener impacto en la vida humana, en la calidad de vida, en el medio ambiente y en la pluralidad étnica, religiosa y cultural.

c. Emitir recomendaciones sobre cuestiones morales y éticas específicas suscitadas en el desenvolvimiento de la ciencia y la tecnología.

d. Emitir recomendaciones y elaborar estudios sobre temas que le fueran enviados.

e. Promover foros de discusión nacional de cuestiones bioética y para la divulgación de sus estudios, dictámenes y recomendaciones 
Sobre el carácter no vinculante de los informes y difusión pública de sus informes, dictámenes $\mathbf{y}$ actuaciones. Por esencia estas comisiones no producen informes vinculantes. Su fuerza radica justamente en la jerarquía de la fundamentación que reflexiona y no omite en reflejar los consensos máximos o mínimos, así como las divergencias que pueden existir en temas de la complejidad de los que abordará. Los informes son insumos para contribuir a la construcción de la opinión y el juicio de la ciudadanía. Debe ser un reflejo de su diversidad. Su difusión amplia a la ciudadanía resulta en beneficio de la sociedad en su conjunto.

Sobre su composición. Como hemos expresado el "rigor lógico, el conocimiento profundo y el juicio sólido" son tres de las cualidades básicas que deben tener los integrantes de este tipo de Comisiones Nacionales lo que se habrá de reflejar en los informes a emitir. La diversidad es la esencia de la composición sin perjuicio de las áreas disciplinarias lo que en ningún caso correspondería fueran todos de una. Por su parte la experiencia y el conocimiento de la diversidad cultural del país es un elemento que fortalecerá el rol de los integrantes máxime si se han integrado instancias de similares características basadas en la importancia de la reflexión y el consenso.

La Comision Nacional de Bioética debe comprender una composición multidisciplinaria, plural y con equidad de género. No existen recetas sobre la cantidad de miembros sugiriéndose un número no menor a 9 destacándose que los mismos deberán actuar a título personal y con total independencia en el ejercicio de sus funciones manteniendo un compromiso de confidencialidad en función de los temas tratados en el seno de la Comisión además de ético. Deberán ser personas con amplio conocimiento de la realidad social y cultural del país, deberán acreditar ante la opinión pública formación en bioética y/o otras especialidades afines con los problemas éticos a abordarse en función de las competencias asignadas y su experiencia en actividades culturales, comunitarias y sociales así como integración de ámbitos de reflexión y participación vinculada a la ética.

Sobre el proceso de selección y designación. No existe un modelo ideal. Sin embargo en todos los casos la designación realizada por las máximas autoridades de la República proyectan su labor. La objetividad de la Comisión se basará en los requisitos esenciales que legitiman la integración y la gestión: interdisciplinariedad, pluralidad, independencia y equidad de género. La publicidad de las candidaturas y la transparencia de su elección serán sus bastiones, y reflejarán las garantías de la Comisión Nacional como herramienta de la democracia abocada a la construcción de una reflexión respetuosa que ilustre la diversidad de pensamientos e ideas de la sociedad en su conjunto. 
Como se ha señalado en anteriores intervenciones públicas se entiende que un posible proceso de selección comprende la presentación de candidaturas a título individual. Por su parte se debería prever que las asociaciones científicas, colegios de profesionales y universidades públicas y privadas, así como también organizaciones de la sociedad civil puedan apoyar la presentación de candidatos sin perjuicio de lo cual, los candidatos o miembros electos no representarán a la institución, asociación u organización que los postula y actuarán con total independencia atento a la previsión legal. Aspecto no menor que asegura la independencia y previene posibles o eventuales conflictos de interés.

Se entiende que la selección debería llevarse a cabo por una Comisión abocada a analizar la referida integración en atención a los perfiles de los candidatos y en cumplimiento de los requisitos de interdisciplinariedad, pluralidad y género establecidos en la presente ley. La Comisión de selección debe contar con una integración plural y asegurar la participación de representantes de la Asamblea General del Poder Legislativo, Poder Ejecutivo, Congreso de Intendentes y Poder Judicial.

Sobre la actuación de los miembros y presupuesto. Se considera que la labor de los miembros de la Comisión es por esencia honoraria. Entre otros aspectos, esta previsión de carácter honorario, contribuye a prevenir y alejar posibles conflictos de interés. Los que se entiende que aún observándose, deberían expresamente advertirse y ser objeto de abstención del miembro involucrado en el mismo.

La independencia externa e interna debe ser su regla. Sin perjuicio de ello resulta vital prever y asegurar un presupuesto que permita consolidar la labor y proyectar las funciones ejecutivas y la debida logística y administración lo que resulta primordial, a los efectos que le permita actuar con la independencia necesaria. De ahí la importancia de que se prevea la creación de una secretaría técnica - administrativa y se otorgue una sede administrativa, además de aporte presupuestal a los efectos logísticos y de funcionamiento. La Secretaría contribuirá a coordinar, organizar y proyectar las acciones de la Comisión Nacional de Bioética. Se sugiere que la misma incluya recursos humanos con la debida formación en bioética y/o otras especialidades afines con los problemas éticos.

En relación a la actuación se sugiere la posibilidad de constituir sub comisiones para el estudio específico de algunos temas pudiendo invitar a participar dentro de éstas a expertos de reconocida solvencia sobre la temática específica a abordar así como también representantes de las asociaciones científicas, academia y/o sociedad civil además de expertos internacionales en Bioética y/o otras especialidades afines con los problemas éticos. La conformación será acordada por los miembros de la Comisión resolviéndose por mayoría su concreción. 
Debe preverse que la Comisión esté facultada para elaborar un reglamento de funcionamiento que defina los aspectos puntuales de la gestión se entiende deben ser articulados en el seno de la Comisión siempre observando los principios básicos incluidos en la ley. El detalle exhaustivo de estos aspectos formales en el texto legal consideramos que puede obstaculizar la flexibilidad y la armonía misma que requiere la actuación de la CNB.

Sobre su rol preceptivo de asesoramiento parlamentario. Como hemos señalado esta comisión tiene como cometido sustancial aportar insumos que desde la diversidad y la fortaleza del contenido, puedan brindar pautas o guías, así como también elementos y consideraciones sobre el tema en discusión, a los representantes nacionales en su labor específica de legislar. Destacamos entonces que la obligatoriedad de solicitar un dictamen técnico a la Comisión Nacional de Bioética fortalece y proyecta la discusión parlamentaria y en definitiva hace realidad la incorporación de la reflexión bioética en el recinto parlamentario. Sin perjuicio del carácter no vinculante de sus dictámenes. Por ende es de especial relevancia que se prevea que el Poder Legislativo a través de sus respectivas comisiones solicite dictamen a la Comision Nacional de Bioética al momento de analizar proyectos de ley sobre las cuestiones bioéticas. El dictamen emitido por la Comisión no será vinculante como se ha señalado.

Sobre los vínculos institucionales e internacionales. Se trata de un aspecto no menor. La sub utilización y sub información que existe en la actualidad de la labor de órganos como el Comité Internacional de la UNESCO y otros en el ámbito internacional es una fuerte carencia constatable en la actualidad con consecuencias que van en desmedro de la actuación de las asociaciones científicas, colegios de profesionales, la academia, organizaciones de la sociedad civil, y la población en general a quien podría de alguna forma aportarse también información. Conviene a los efectos de fortalecer la inclusión de esta previsión revisar los informes emitidos por el Comité Internacional creado en virtud de la Declaración del Genoma Humano y los Derechos Humanos en el seno de la UNESCO. La propuesta radica en vincular efectivamente la acción de la Comisión con estos ámbitos para "observar" y a la vez relevar insumos de actualidad mundial en relación a los dilemas y debates desde la diversidad cultural universal. Así también fortalecer el vínculo con otras comisiones de la región.

Se sugiere entonces que la Comisión Nacional de Bioética se vincule a través del Ministerio de Relaciones Exteriores con el Comité Internacional de Bioética de la UNESCO debiendo participar en calidad de observador de sus sesiones anuales. Que se incluya dentro de sus competencias la difusión en forma permanente de los insumos que emanen de dicho Comité Internacional en carácter de informes en los ámbitos 
especializados así como también a la población en general de una manera comprensible y sintética. Estos cometidos contarán con el apoyo del Ministerio de Relaciones Exteriores y la Delegación Uruguaya ante UNESCO.

Así también que sea la Comisión Nacional de Bioética la que represente a Uruguay en las instancias regionales e internacionales que se llevan a cabo con otras Comisiones de la misma naturaleza jurídica.

Sobre la coordinación con comisiones o comités de bioéticas institucionales. El rol de esta Comisión Nacional de Bioética como referente público del abordaje de los problemas de la ética constituye uno de sus objetivos. Así también que la Comisión Nacional se constituya en referente de las comisiones de bioética institucionales. Se trata de que las comisiones de bioética que deben operar en el seno de los centros asistenciales atento al mandato legal (ley 18335 y decreto reglamentario) tengan en los hechos y en la práctica una actuación activa con las características de asesorar internamente, aportar elementos que puedan promover guías o pautas éticas y educar tanto al personal como a los usuarios y pacientes. La realidad demuestra que a pesar de estar creadas y sin perjuicio de la actividad destacada de algunas de ellas, un importante número de estas comisiones permanecen huecas, siendo desconocida su actuación a nivel de los usuarios y pacientes del sistema y sub utilizada por los propios prestadores del servicio asistencial. Por ello se entiende que el rol de la Comision Nacional puede potenciar y multiplicar la implementación activa de la labor de las comisiones de esta naturaleza, creadas en el seno de cada institución y con ello aportando elementos para que la ciudadanía se acerque a la comprensión de los principios bioéticos y su incidencia en su vida y salud.

Atento a lo expuesto se sugiere prever que la Comisión Nacional de Bioética mantenga una comunicación fluida con las Comisiones de Bioética creadas en el ámbito institucional, llevando a cabo una reunión anual con representantes de todas las comisiones de bioética constituidas en los centros asistenciales y de emergencia móvil; con la participación de la academia, asociaciones científicas, colegios profesionales y organizaciones de la sociedad civil vinculadas a la temática, así como expertos internacionales, la que podrá ser de carácter presencial o virtual. 


\section{BIBLIOGRAFÌA CONSULTADA.}

Sobre la Dignidad y los principios: Análisis de la Declaración Universal sobre Bioética y Derechos de la UNESCO. "ARTÍCULO 19, comités de ética", (CASADO, M. Coordinadora) Cívitas, 2009. Disponible en:https://obtienearchivo.bcn.cl/obtienearchivo?id=document os/10221.1/53026/1/252153.pdf Fecha de consulta: 6 febrero 2021.

DE LECUONA, Itziar. La regulación española de los Comités de Etica ylas novedades introducidas por la nueva ley de investigación biomédica. Disponible en: file://C:/Users/Usuario/Downloads/1470128167-1-SM.pdf Fecha de consulta: 3 febrero 2021

Comités y las Comisiones Nacionales de Bioética en América Latina y el Caribe. Situación actual, propuestas y desafíos. (MAGLIO, Ignacio, Coordinador). Editado por UNESCO y Universidad del Bosque. Colombia, 2018.

Disponible en: https://www.unbosque.edu.co/sites/default/files/2018-09/Los\%20comit \%c3\%a9s\%20y\%20 las\%20comisiones.pdf Fecha de +consulta: 6 febrero 2021.

Diccionario Latinoamericano de Bioética. (TEALDI, Juan Carlos, Coordinador). Editado por UNESCO y la Universidad Nacional de Colombia. Colombia, 2008. Disponible en: https://unesdoc.unesco.org/ ark:/48223/pf0000161848 Fecha de consulta: 8 febrero 2021.

Guía No. 1. Creación de Comités de Bioética. UNESCO. Francia, 2006. Disponible en: https:/unesdoc. unesco.org/ark:/48223/pf0000139309_spa Fecha de consulta: 6 febrero 2021.

Guía No. 2. Funcionamiento de los Comités de Bioética. Francia, 2006. Disponible en: https:/unesdoc. unesco.org/ark:/48223/pf0000147392_spa Fecha de consulta: 6 febrero 2021.

Guía No. 3.Capacitación de Comités de Bioética. UNESCO. Francia, 2007. Disponible en: https://unesdoc. unesco.org/ark:/48223/pf0000150970_spa Fecha de consulta: 6 febrero 2021.

GROS ESPIELL, Héctor. Etica, Bioética y Derecho. Editorial Temis. Bogotá, Colombia, 2005.

ROTONDO, Teresita. Aportes para una historia de la Bioética en el Uruguay. (en imprenta). 